\title{
Comparing Privacy Attitudes of Knowledge Workers in India and the U.S.
}

\author{
Sameer Patil ${ }^{1}$, Alfred Kobsa ${ }^{1}$, Ajita John ${ }^{2}$, and Doree Seligmann ${ }^{2}$ \\ 1 Department of Informatics \\ University of California, Irvine \\ Irvine CA 92617 USA \\ \{patil, kobsa\}euci.edu \\ 2 Collaborative Applications Research \\ Avaya Labs \\ Basking Ridge, NJ 07920 USA \\ \{ajita, doree\}eavaya.com
}

\begin{abstract}
Enterprises are increasingly deploying social computing platforms, such as blogs, shared bookmarks, and social networks, to support the interpersonal interactions and knowledge work practices of their employees. These technologies hold great promise for supporting distributed collaboration by enabling greater communication and fostering awareness among colleagues. Promotion of awareness and communication, however, faces an inherent tension with desires for privacy. Additionally, differences in privacy expectations and practices between parties who interact through these systems could lead to misunderstandings and miscommunication. This paper reports on a study of geographically distributed knowledge workers collaborating on a software development project in a multinational corporation. Contrary to expectations, we found that knowledge workers in India expressed higher privacy concerns compared with their colleagues in the US. We are currently analyzing the data from field visits, interviews and questionnaire responses, to uncover plausible explanations for the observed differences.
\end{abstract}

\section{Introduction}

One of the major forces driving societal transformation in the past decade has been the penetration of technology in even the most mundane spheres of daily life. Computationally powerful mobile communication devices like the iPhone are accelerating the trend towards pushing computing off-the-desktop and making it available anytime, anywhere. Instant Messaging (IM), social networking, and blogging have revolutionized interpersonal interaction practices, both private and public. Along the same lines, enterprises engaged in knowledge work are increasingly adopting and adapting information systems that leverage such social computing platforms in order to facilitate interactions and collaboration among knowledge workers [1].

These systems have also led to enormous changes in the scope and nature of collaboration in knowledge work. Traditionally, people who worked together were colocated, and collaborated face-to-face. In recent years, however, networked information 
and communication systems have made it possible for collaborative activities to be distributed physically as well as temporally. Today, it is not uncommon to find teams comprised of members distributed across different continents. Moreover, a significant portion of knowledge work is being transferred from North America and Europe to countries such as India, China and the Philippines.

A large part of the appeal of these technologies for supporting distributed collaborative work lies in their potential for enabling greater communication (work-related as well as social) and fostering awareness among colleagues. It is well known that awareness and communication are crucial for raising the effectiveness and efficiency of collaborative endeavors [2]. Promotion of awareness and communication, however, faces an inherent tension with desires for privacy [3]. Inability of individuals to achieve sufficient privacy could lead to a reduction in their efficiency. For example, having less privacy than desired could lead to increased inopportune interruptions whereas having more privacy than necessary may create extra burden on others to solicit information useful for structuring their work. As a result, it is critical that interaction systems provide effective ways for users to reconcile their privacy desires with awareness needs.

Additionally, differences in privacy expectations and practices between parties who interact through these systems may lead to misunderstandings and miscommunication. Therefore, awareness systems need to accommodate such variations in preferences and practices. Moreover, given the increasingly global nature of collaborative projects, it is important to investigate whether national differences influence these issues. Towards this end, we report on a study of geographically distributed knowledge workers collaborating on a software development project in a multinational corporation. Specifically, we compare the privacy concerns of knowledge workers in the U.S. and India.

\section{Related Work}

Initial privacy-related findings were primarily noted "on the side" in studies aimed at evaluating experiences with the awareness aspects of interaction systems (see e.g., [4-6]). Recently, studies have started targeting privacy as the primary object of investigation (see e.g., [7-9]) Patil and Kobsa [10] provide a more detailed discussion of these studies. Additionally, researchers have examined the role played by the physical characteristics of the workplace in employee work practices (see e.g., [11-13]). Most of the studies, however, were conducted in North America or Europe. Our study broadens the reach by seeking to uncover the differences between privacy attitudes of knowledge workers in India and the U.S. In contrast to prior work, our study aimed at investigating these issues across all information systems that are utilized in collaborative work practice rather than focusing on a specific system.

\section{Setting and Methodology}

We studied a software development project (henceforth Project X) at a large multinational telecommunications company. At various stages, Project $\mathrm{X}$ included anywhere between 80 to 130 contributors spread across at least five different locations: four in the U.S. (in three different time zones) and one in India. To avoid biasing the participants, 
the goal of the study was advertised as an investigation of collaborative work practices. Given the lack of a universal definition for privacy, we did not confine participants to a specific view of privacy. Therefore, rather than imposing a definition of privacy, we asked them to explain what "privacy" meant to them in the context of their work and work practices. This enabled us to uncover the various contextual meanings of privacy for the participants, and the associated behaviors and practices aimed at satisfying privacy needs. We used the following methods:

Non-participant observation: We started with non-participant observation of the meetings of the Project X management team.

Site visits: During the first phase of the study, the author was based at a Project $X$ site on the East Coast of the U.S., and visited three of the other sites, in mid-U.S., on the U.S. West Coast, and in India, each for about a week. At every location, interviews were conducted with project members (see below), and site-specific factors such as architecture, layout, work practices and culture were documented. We also interviewed project contributors from the remaining U.S. site - also located on the East Coast while they were visiting the first-mentioned site.

Semi-structured interviews: At each visited site, we conducted semi-structured interviews lasting about 90 minutes. The questions were divided into three themes: work practices, awareness and privacy needs, and desired enhancements to collaborative tools. In total, we interviewed fifty-two project members across the five sites.

Online questionnaire: Based on key insights from the above activities, we formulated an online questionnaire. Additionally, we used questions from the literature to measure privacy attitudes and practices in the domain of consumer privacy $[14,15]$, and collected demographic information. The questionnaire was distributed to all individuals involved with Project $\mathrm{X}$ at that time (roughly 125). We obtained 90 valid responses (response rate of $74 \%$ ) which included responses from 30 of our original 52 interviewees.

\section{Findings}

We divided the questionnaire respondents into two groups: those who worked at the India site (India) and those at any U.S. site (US). Group US consisted of individuals of several nationalities (including some Indians). Since national culture affects privacy considerations, we excluded from Group US those who had not lived in the U.S. for at least five years. Even though Group India consisted wholly of Indian nationals, for the same reason, we excluded from Group India those who had lived in the U.S. for longer than five years before returning to India. The final dataset after the filtering comprised of 52 individuals in Group US and 35 in Group India.

Based on prior research [16], we expected lower privacy concerns from Group India than Group US. True to these expectations, we found that Group US reported significantly higher engagement than Group India in protecting the privacy of personal information from third parties $(\mathrm{p}<0.01)$. Specifically, these practices include: inquiring about policies regarding the handling of their personal information, refusing to provide personal information, or refusing to make purchases if the organization does not protect personal information adequately, asking to remove one's personal information or pro- 
hibiting the sale of one's personal data, and checking to see which personal information about oneself had been collected by an organization.

The questionnaire also asked respondents to rate how concerned they were about privacy with regard to various categories of people with whom they interact in the course of their work (on a 7-point Likert scale from "completely unconcerned" to "extremely concerned"). These groups were: team members at the local site (excluding the manager), team members at remote sites (excluding the manager), manager, Project $\mathrm{X}$ peers outside of one's team at the local site, Project X peers outside of one's team at remote sites, upper management, company employees at the local site (but not working on Project X), company employees at remote sites (but not working on Project X), subordinates (if applicable), and system and IT administrators and support staff.

To our surprise, we found that those in Group India expressed higher privacy concerns than those in Group US. These differences are statistically significant for those categories of contacts that one typically works closely with, viz. local and remote team members, manager, and local and remote Project X members. Although the differences for the other categories are not statistically significant, the privacy concerns of Group India are still higher except in the case of subordinates.

In a similar vein, on a scale of 1 (strongly disagree) to 7 (strongly agree), those from Group India express a higher desire for privacy management tools (India 5.1, US $4.27, \mathrm{p}<0.033)$. Group India also indicates a slightly higher willingness to spend time in configuring such tools if they offer better privacy management (India 4.9, US 4.5) although this difference is not statistically significant.

We also compared the reported attitude of the two groups towards interruptions, a factor linked frequently to privacy in the literature [17]. Despite expressing comparatively higher privacy concerns, Group India found interruptions useful to a much larger extent than Group US on a scale of 1 to 7 (India: 4.80, US: 3.52, p < 0.00001). On the other hand, Group US, despite expressing comparatively lower privacy concerns, found interruptions disruptive to a much larger extent (India: 4.26 US: $5.10 \mathrm{p}<0.015$ ).

\section{Discussion}

We are currently analyzing our data in depth in order to uncover explanations for the surprisingly higher privacy concerns of Group India. Preliminary analysis points to the following as plausible underlying factors:

- Physical characteristics of the workplace

- Nature of interpersonal relationships

- Conceptualization of privacy

- Intra-team comptition

- Management style and hierarchy

\section{Conclusion}

We found differences in the privacy concerns of knowledge worker collaborators in India and the U.S. To our surprise, we found that knowledge workers in India expressed 
higher privacy concerns compared with their colleagues in the US. We are currently engaged in a deeper analysis of our field data to uncover explanations for the differences. Due to the commonalities across the Indian software industry, we suspect that these findings will apply more generally. The findings also highlight that awareness systems need to accommodate interactions between populations with varied preferences and practices. We hope that this work will pave the way for future studies of cultural contexts outside of North America and Western Europe.

\section{Acknowledgments}

The authors acknowledge Mihir Mahajan and Tapan Khopkar for their comments. The authors also thank all participants of the study for their time. This research has been supported by NSF Grant Nos. 0205724 and 0808783.

\section{References}

1. FaceTime Communications: The Collaborative Internet: Usage Trends, End User Attitudes and IT Impact (2008)

2. Dourish, P., Bly, S.: Portholes: Supporting Awareness in a Distributed Work Group. In: CHI '92: Proceedings of the SIGCHI Conference on Human Factors in Computing Systems, New York, NY, USA, ACM (1992) 541-547

3. Hudson, S.E., Smith, I.: Techniques for Addressing Fundamental Privacy and Disruption Tradeoffs in Awareness Support Systems. In: CSCW '96: Proceedings of the 1996 ACM Conference on Computer Supported Cooperative Work, New York, NY, USA, ACM (1996) 248-257

4. Palen, L.: Social, Individual and Technological Issues for Groupware Calendar Systems. In: CHI '99: Proceedings of the SIGCHI Conference on Human Factors in Computing Systems, New York, NY, USA, ACM (1999) 17-24

5. Lee, A., Girgensohn, A., Schlueter, K.: NYNEX Portholes: Initial User Reactions and Redesign Implications. In: GROUP '97: Proceedings of the International ACM SIGGROUP Conference On Supporting Group Work, New York, NY, USA, ACM (1997) 385-394

6. Herbsleb, J.D., Atkins, D.L., Boyer, D.G., Handel, M., Finholt, T.A.: Introducing Instant Messaging And Chat In The Workplace. In: CHI '02: Proceedings of the SIGCHI Conference on Human Factors in Computing Systems, New York, NY, USA, ACM (2002) 171-178

7. Lederer, S., Mankoff, J., Dey, A.K.: Who Wants to Know What When? Privacy Preference Determinants in Ubiquitous Computing. In: $\mathrm{CHI}$ '03: CHI '03 Extended Abstracts on Human Factors in Computing Systems, New York, NY, USA, ACM (2003) 724-725

8. Olson, J.S., Grudin, J., Horvitz, E.: A Study of Preferences for Sharing and Privacy. In: CHI '05: CHI '05 Extended Abstracts on Human Factors in Computing Systems, New York, NY, USA, ACM (2005) 1985-1988

9. Patil, S., Lai, J.: Who Gets to Know What When: Configuring Privacy Permissions in an Awareness Application. In: CHI '05: Proceedings of the SIGCHI Conference on Human Factors in Computing Systems, New York, NY, USA, ACM (2005) 101-110

10. Patil, S., Kobsa, A.: Privacy Considerations in Awareness Systems: Designing with Privacy in Mind. In Verlag, S., ed.: Awareness Systems: Advances in Theory, Methodology and Design. Panos Markopoulos and Boris de Ruyter and Wendy Mackay (2009)

11. Stokols, D., Clitheroe, C., Zmuidzinas, M.: Qualities of Work Environments That Promote Perceived Support for Creativity. Creativity Research Journal 14(2) (2002) 137-147 
12. Haans, A., Kaiser, F.G., de Kort, Y.A.W.: Privacy Needs in Office Environments: Development of Two Behavior-Based Scales. European Psychologist 12(2) (2007) 93-102

13. Birnholtz, J.P., Gutwin, C., Hawkey, K.: Privacy in the Open: How Attention Mediates Awareness and Privacy in Open-plan Offices. In: GROUP '07: Proceedings of the 2007 International ACM Conference on Supporting Group Work, New York, NY, USA, ACM (2007) 51-60

14. IBM: IBM Multi-National Consumer Privacy Survey (October 1999)

15. Westin, A.F.: Harris-Equifax Consumer Privacy Survey 1991. (1991)

16. Kumaraguru, P., Cranor, L.F., Newton, E.: Privacy Perceptions in India and the United States: An Interview Study. In: The 33rd Research Conference on Communication, Information and Internet Policy (TPRC). (2005)

17. Altman, I.: The Environment and Social Behavior: Privacy, Personal Space, Territory, Crowding. Brooks/Cole, Monterey, California (1975) 